\title{
Outcomes of Open Surgery for Popliteal Artery Aneurysms: An 18-Year Experience in a Single Institution in Japan
}

\author{
Yoshihisa Makino, Katsuyuki Hoshina*, Kota Yamamoto, Masaru Kimura \\ Division of Vascular Surgery, Department of Surgery, the University of Tokyo, Tokyo, Japan \\ Email: *traruba@gmail.com
}

How to cite this paper: Makino, Y., Hoshina, K., Yamamoto, K. and Kimura, M. (2018) Outcomes of Open Surgery for Popliteal Artery Aneurysms: An 18-Year Experience in a Single Institution in Japan. World Journal of Cardiovascular Surgery, 8, 51-60.

https://doi.org/10.4236/wjcs.2018.83006

Received: February 6, 2018

Accepted: March 19, 2018

Published: March 22, 2018

Copyright $\odot 2018$ by authors and Scientific Research Publishing Inc. This work is licensed under the Creative Commons Attribution International License (CC BY 4.0).

http://creativecommons.org/licenses/by/4.0/

(c) (i) Open Access

\begin{abstract}
Objective: To elucidate the outcomes of open surgery for popliteal artery aneurysm (PAA) in a single Japanese institution. Materials and Methods: Between 1999 and 2016, 35 patients (44 PAAs) underwent open surgery in our department. The first-line surgical strategy involved the medial approach, with excision of the aneurysm sac and autologous vein bypass grafting. Associations between the outcomes and 1) the surgical management of the aneurysmal sac (aneurysmectomy vs. ligation with bypass) and 2) the preoperative clinical symptoms were evaluated. Results: There were no aneurysm-related deaths during the follow-up period (mean, 4 years; range, 1 - 17.6 years). The 5-year primary, primary-assisted, and secondary patency rates were $67.9 \%$, $72.7 \%$, and $94.5 \%$, respectively. Re-intervention was required for 19 PAAs (14 patients), including two PAAs in patients with suspected Behçet's disease. Patency rates did not differ between the aneurysmectomy and ligation groups. Remnant sac enlargement occurred in 3 cases. Among asymptomatic patients, clinical symptoms did not deteriorate; however, the ankle brachial pressure index decreased $\geq 0.15$ in patients with artery-graft mismatching. Conclusion: Patency rates were good, and our modified ligation with bypass procedure, which involves excising the sac as much as possible, exhibited less risk in terms of remnant sac enlargement compared to that in previous studies.
\end{abstract}

\section{Keywords}

Popliteal Artery Aneurysm, Approach, Bypass, Behçet’s Disease

\section{Introduction}

The treatment strategy for popliteal artery aneurysms (PAAs) differs from that 
for other aneurysms. In cases of PAA, surgery is performed for the purpose of limb salvage, as well as for extending the life [1] [2] [3]. Arterial thrombosis due to a PAA and subsequent limb loss is detrimental to the patient's quality of life; thus, PAAs should be treated when identified, irrespective of the aneurysm diameter [1] [4].

PAA treatment is also unique in terms of the variety of available surgical procedures, which depend on the aneurysm location, size, and morphology. There are two main surgical approaches (medial and posterior), the selection of which remains controversial [5] [6]. Although the superiority of the posterior approach has been recently reported [7], some surgeons prefer the medial approach, as it does not require patients to be in the prone position during surgery and has a reduced risk of dissection-related neurological complications [6]. Given that our first-line therapy is the medial approach, we recognize the downsides to this approach: the aneurysm sac cannot be completely excised, particularly with a large PAA, and the anastomosis of the distal site may be difficult. In addition, remnant sac enlargement is a troublesome adverse event that may occur during the follow-up period.

The mortality and morbidity rates in the surgical intervention of PAAs are considered excellent [8] [9]. However, to improve outcomes, the impact of the patient's background and selected procedural method on re-intervention and adverse events should be analyzed in detail. Although a previous study indicated that race (African-American) is a risk factor associated with mortality and morbidity [10], additional studies analyzing race are lacking, and there are few reports on PAAs in Asia. The purpose of the present study was to elucidate the outcomes of open surgery, using our own methodology, in patients of the same race with PAAs, in a single institution. In addition, we evaluated the associations between the outcomes and 1) the preoperative clinical symptoms, and 2) the selected surgical procedures (aneurysmectomy vs. ligation with bypass).

\section{Materials and Methods}

Patients and surgical strategy. This retrospective study adhered to the guidelines of the research ethics committee of our institution, and was comprehensively approved by the institutional Research Ethics Committee of The University of Tokyo Hospital [No. 3316 (3)]. All patients provided written informed consent for participation.

Patients who underwent surgery for a PAA in our department between January 1999 and April 2016 were included. Our treatment strategy for PAAs was determined after the 1990s, and it was difficult to obtain computed tomography images and detailed descriptions in the clinical records of older cases. Thus, we selected patients from 1999 and onward. Surgical intervention was performed when the PAA was $>2 \mathrm{~cm}$ in diameter or the focal dilatation was $>1.5$ times the expected normal diameter. Surgery was not performed in patients with a PAA with a completely thrombosed sac, but no symptoms of critical limb ischemia 
due to sufficient collateral vessels.

In our first-line surgical strategy the medial approach was used, with as much excision as possible of the aneurysm sac from the above-knee incision, accompanied by autologous vein bypass grafting. We sometimes selected a prosthetic graft in cases exhibiting a large discrepancy between the native popliteal artery and the autologous vein graft. Patients underwent aneurysm sac excision (aneurysmectomy; Group A) or ligation with bypass and no/incomplete excision of the aneurysm sac (Group L). The procedure selection depended on the size and location of the aneurism.

Outcomes and definitions. Patients were followed-up every 6 months at outpatient clinics; graft patency was assessed via graft palpation, ultrasonography, and contrast-enhanced computed tomography. Primary patency was defined as the maintenance of blood flow through the bypass graft after implantation, without interventions. Primary-assisted patency was defined as the maintenance of blood flow through the bypass graft after implantation, regardless of re-interventions. Secondary patency was defined as the maintenance of blood flow through the bypass graft, regardless of re-interventions, following a total occlusion.

Adverse events requiring re-intervention were recorded and included graft failure, anastomotic failure, graft infection, and remnant sac enlargement. The ankle brachial pressure index (ABI) was assessed in 11 cases and the change in $\mathrm{ABI}$ was calculated using the following equation: preoperative $\mathrm{ABI}$-postoperative $\mathrm{ABI} /$ preoperative ABI.

Statistical analysis. Statistical analyses were performed using JMP ${ }^{\circledR} 9.0$ software (SAS Institute Inc. Cary, NC, USA). Continuous data are expressed as mean \pm standard deviation. Group differences were evaluated using the Student's t-test for continuous variables and the chi-squared test for categorical variables with cell sizes $>4$. Group differences in the patency rates were evaluated using the Kaplan-Meier method with the log-rank test. A p-value $<0.05$ was considered statistically significant.

\section{Results}

A total of 35 patients (44 PAAs) were included. The mean age of the patients was $64.8 \pm 13.7$ years, and the mean PAA diameter was $32.3 \pm 14.2 \mathrm{~mm}$. Four patients had notable clinical histories: three patients had suspected Behçet's disease, but did not completely fulfill the criteria; and one patient was diagnosed with Klippel-Trenaunay syndrome. A total of 50 concomitant aneurysms were observed in 14 patients. Aneurysms were located in the thoracic aorta (3 cases), abdominal aorta (6 cases), common iliac artery (16 cases in 9 patients), internal iliac artery (10 cases in 6 patients), femoral artery (14 cases in 9 patients), and subclavian artery (1 case).

Outcomes and re-intervention. There were no aneurysm-related deaths during the follow-up period. The mean follow-up period was 3.4 years (range, 1 
- 17.6 years). The 5-year primary, primary-assisted, and secondary patency rates were $67.9 \%, 72.7 \%$, and $94.5 \%$, respectively (Figure 1 ).

Surgical re-intervention was required for 19 PAAs in 14 patients. The causes of re-intervention included amputation (2 cases); graft infection (2 cases); graft failure including stenosis, occlusion, and aneurysm (7 cases); anastomotic failure (5 cases); and remnant sac enlargement (3 cases). Two patients with suspected Behçet's disease had poor outcomes; one patient died of a thoracic aortic dissection at 8 months postoperative, and one patient underwent re-intervention twice (for a graft infection at 7 months and a graft aneurysm at 11 years postoperative).

Surgical management of the aneurysmal sac (aneurysmectomy vs. ligation with bypass) and outcomes. In total, there were 18 cases in Group A and 26 cases in Group L. Group differences in patient background characteristics, bypass inflow and outflow, and patency were evaluated (Table 1). The mean PAA diameter in Group A was larger than that in Group L (38.7 \pm 19.1 vs. $28.3 \pm 10.6$ $(\mathrm{mm}), \mathrm{p}=0.047)$. The comorbidities, operative time, blood loss and in-hospital duration after the operation were not statistically different between the groups. The bypass inflow and outflow data are shown in Figure 2. In most cases, bypass grafting was from the superficial artery to the distal popliteal artery (Figure 2). The site selection was simpler in Group A compared to that in Group L; it was difficult to find an appropriate site in Group L, as the remnant sac occupied the space for the surgical procedures. There were no significant group differences in the patency rates. The primary, primary-assisted, and secondary patency rates for Group A vs. Group L were $66.4 \%$ vs. $67.1 \%$ ( $\mathrm{p}=0.97$ ), $66.4 \%$ vs. $75.5 \%$ ( $\mathrm{p}=$ 0.47 ), and $94.4 \%$ vs. $94.4 \%$ ( $\mathrm{p}=0.78$ ), respectively (Figure 3 ).

Preoperative symptoms and outcomes. There were 27 asymptomatic patients, 7 patients with intermittent claudication, and 11 patients with pain at rest

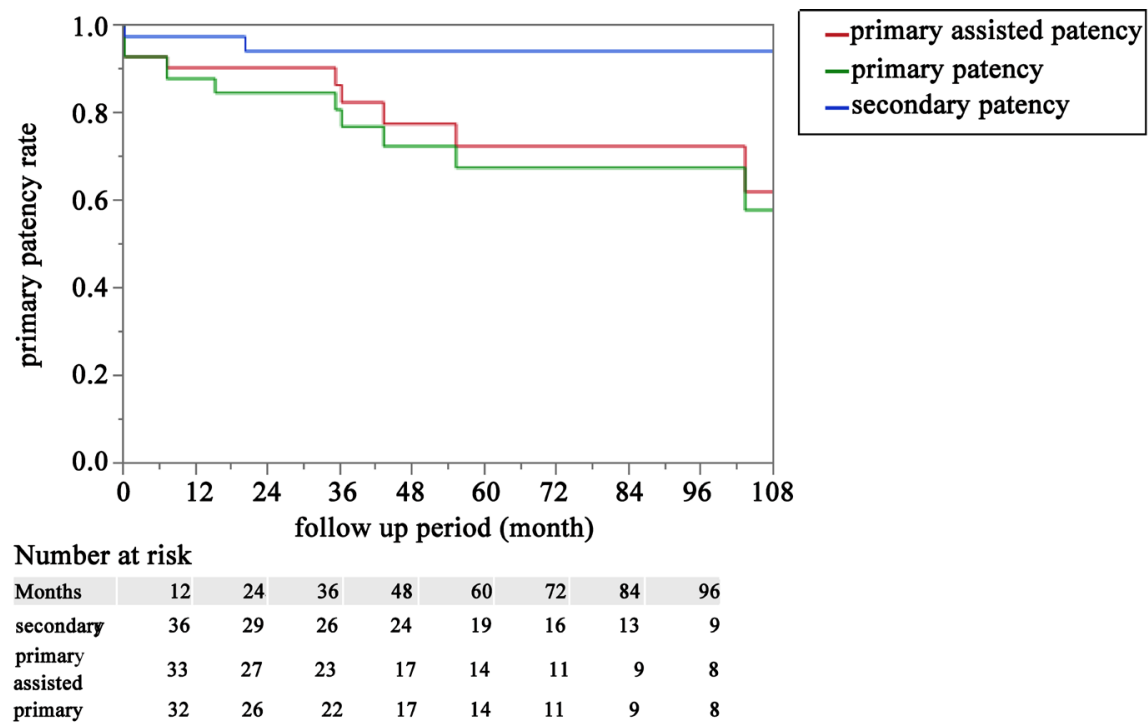

Figure 1. Patency rates in surgically treated popliteal artery aneurysms. Primary, assisted-primary, and secondary patency rates are shown in the Kaplan-Meier curves. 
Table 1. Comparisons of the patients' background characteristics between Group A (aneurysmectomy) and Group L (ligation).

\begin{tabular}{|c|c|c|c|}
\hline Variables & Group A $(n=18)$ & Group L $(n=26)$ & $P$ value \\
\hline Age & $69.5 \pm 12.8$ & $64.1 \pm 10.0$ & 0.14 \\
\hline Male sex (\%) & $13(72 \%)$ & $19(73 \%)$ & 1.00 \\
\hline \multicolumn{4}{|l|}{ Aneurymal diameter } \\
\hline (Antero-posterior) (mm) & $38.7 \pm 19.1$ & $28.3 \pm 10.6$ & 0.05 \\
\hline \multicolumn{4}{|l|}{ Comorbidities } \\
\hline Hypertension & $13(72 \%)$ & $18(69 \%)$ & 1.00 \\
\hline Ischemic heart disease & $1(5 \%)$ & $8(30 \%)$ & \\
\hline Cerebrovascular disease & $4(22 \%)$ & $7(26 \%)$ & \\
\hline Diabetes mellitus & $2(11 \%)$ & $4(15 \%)$ & \\
\hline Respiratory failure & $6(33 \%)$ & $6(23 \%)$ & 0.50 \\
\hline Chronic renal failure & $5(27 \%)$ & $6(23 \%)$ & 0.73 \\
\hline Smoking (ex or current) & $10(55 \%)$ & $19(73 \%)$ & 0.33 \\
\hline Anti-platelet or coagulant drug use & $9(50 \%)$ & $16(62 \%)$ & 0.54 \\
\hline \multicolumn{4}{|l|}{ Operation } \\
\hline Operative time (min) & $276 \pm 114$ & $310 \pm 87$ & 0.28 \\
\hline Intraoperative blood loss (ml) & $253 \pm 225$ & $193 \pm 120$ & 0.31 \\
\hline Postoperative in-hospital duration (days) & $19.4 \pm 11.1$ & $22.7 \pm 13.3$ & 0.37 \\
\hline Approach: & 16 & 26 & \\
\hline Posterior & 2 & 0 & \\
\hline \multirow[b]{2}{*}{ Prosthetic graft } & 12 & 24 & \\
\hline & 6 & 2 & \\
\hline
\end{tabular}

Inflow vessels

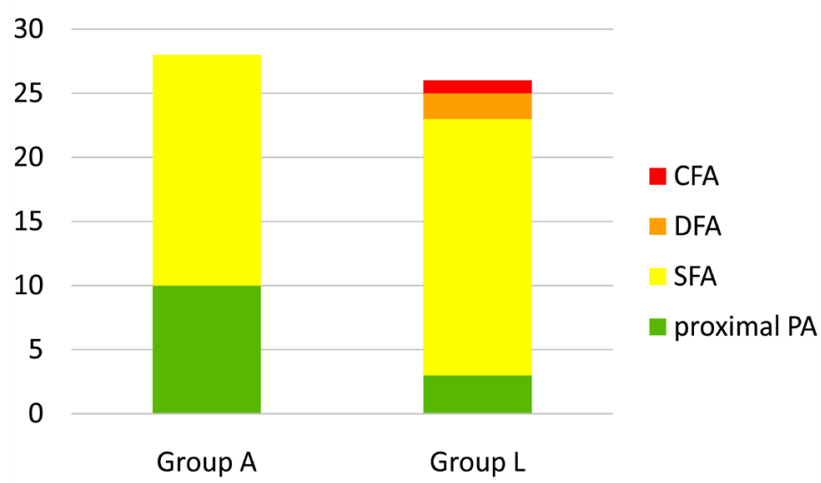

Outflow vessels

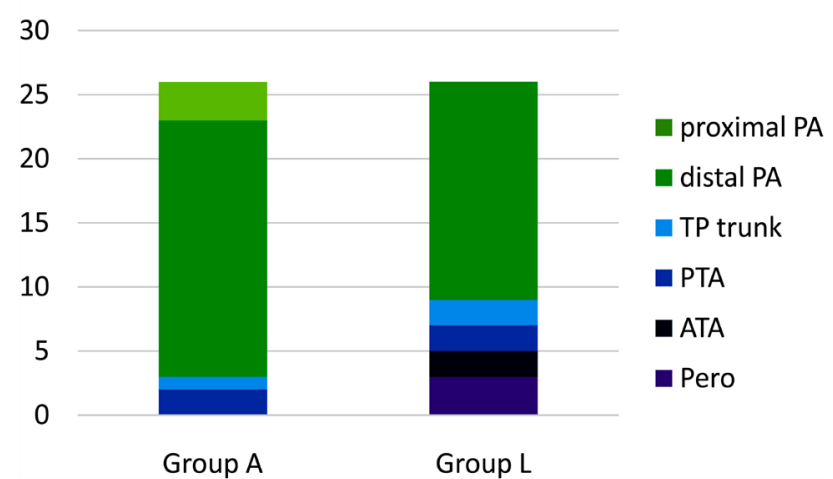

Figure 2. Sites of bypass inflow and outflow in Group A (aneurysmectomy) and Group L (ligation). CFA, common femoral artery; DFA, deep femoral artery; SFA, superficial femoral artery; PA, popliteal artery; TP trunk, tibioperoneal trunk; PTA, posterior tibial artery; ATA, anterior tibial artery; Pero, peroneal artery. 

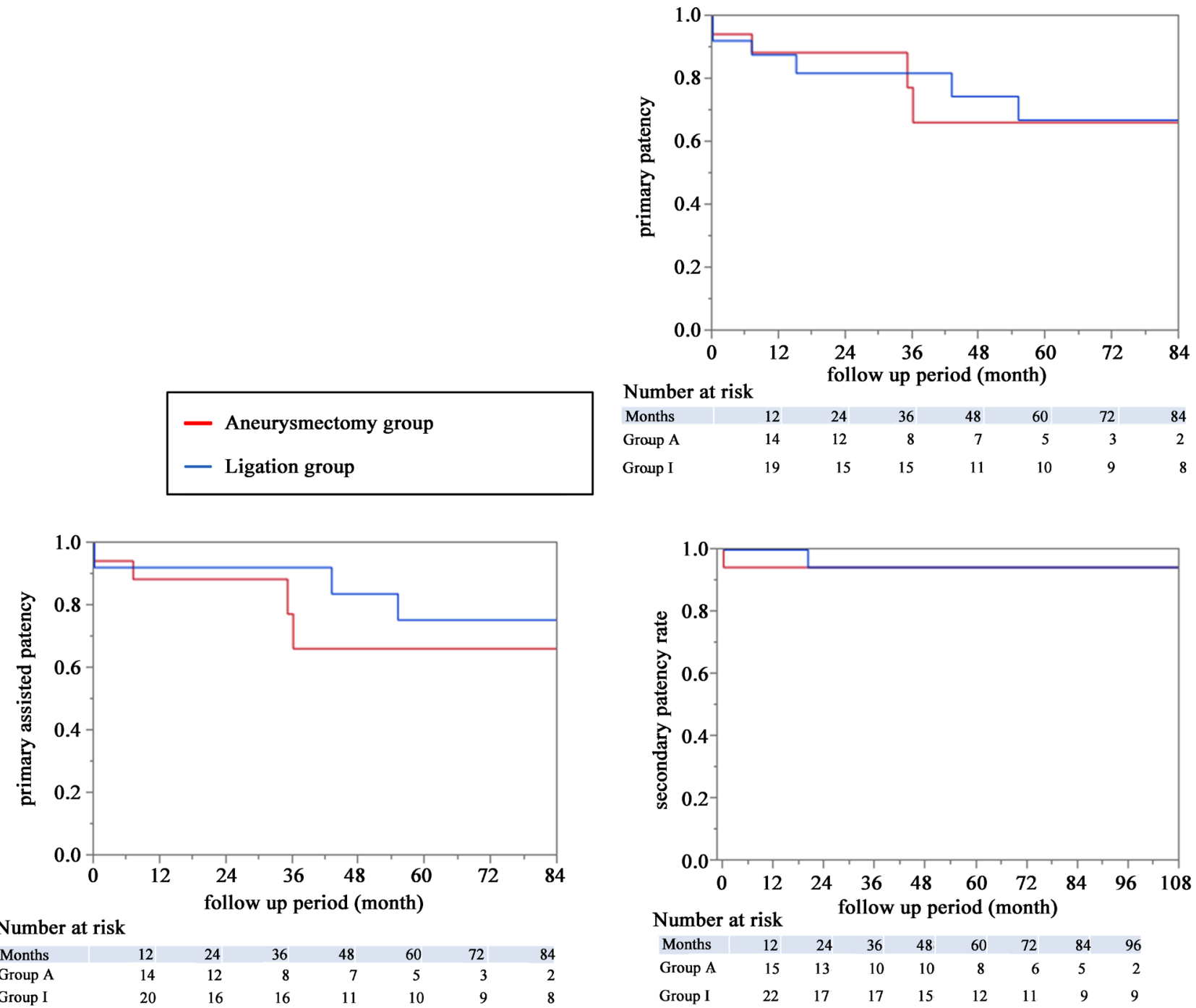

Figure 3. Comparisons of the primary, primary-assisted, and secondary patency rates between Group A (aneurysmectomy) and Group L (ligation).

or tissue loss. Asymptomatic patients did not exhibit any postoperative ischemic symptoms. Claudication improved postoperatively in all 7 patients with intermittent claudication. One major and two minor amputations were performed in the 11 patients with pain at rest or tissue loss. Notably, among the asymptomatic patients, 5 patients exhibited a decrease in the ABI of $\geq 0.15$. All such cases had been bypassed with grafts of $<4 \mathrm{~mm}$ (Figure 4). Furthermore, the mean diameter was significantly smaller $(3.5 \mathrm{~mm})$ in the patients with a decrease in the ABI $\geq 0.15$ compared to that in patients without a decrease in the ABI $\geq 0.15$ (4.8 $\mathrm{mm})(\mathrm{p}=0.007)$.

\section{Discussion}

The outcomes in the present study can be considered acceptable compared to those in the existing literature [8] [9] [11]. Excellent primary patency rates $(\sim 85 \%)$ have been reported in asymptomatic patients [8] [9]; however, the rate 


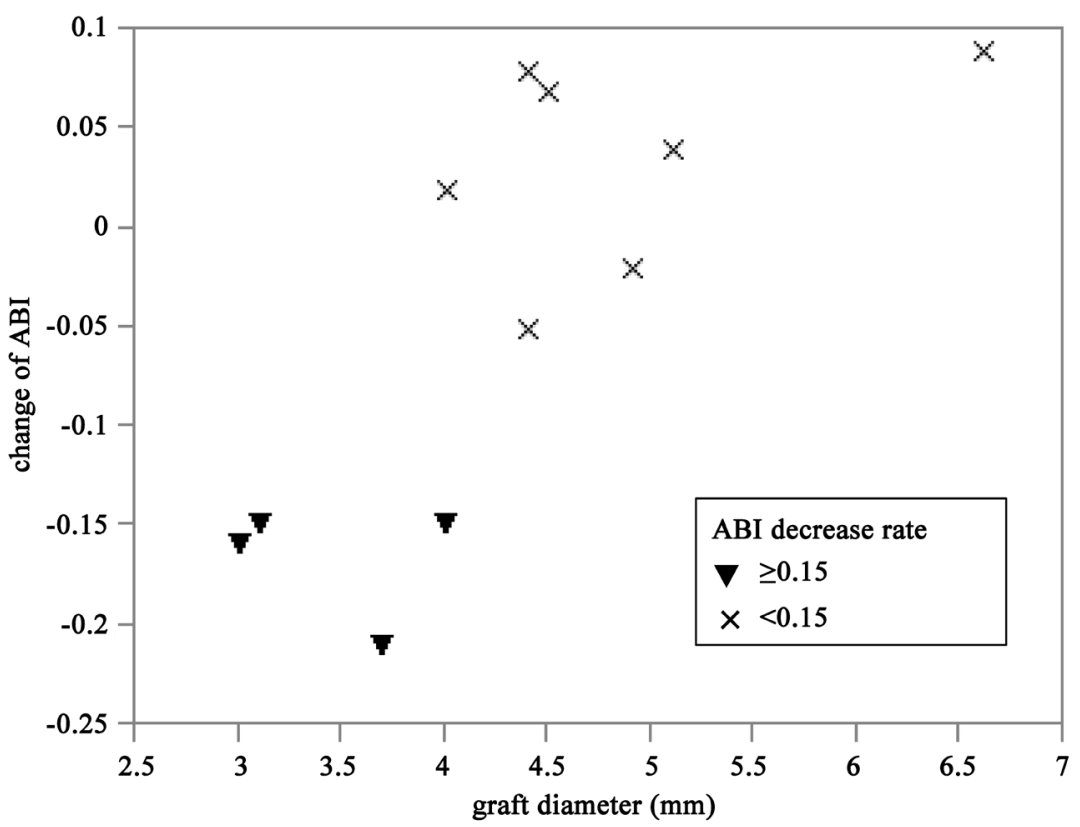

Figure 4. Relationship between the rate of ankle brachial pressure index (ABI) change and graft diameter.

for all patients with PAAs is $\sim 70 \%$ [11] [12], which similar to that in the present study. The secondary patency rate of $94 \%$ observed in the present study is comparable to that in previous reports [8] [11]. Most of the observed adverse events were identified within 1 year postoperative. Infections tended to occur as an early complication, while sac enlargement was detected during long-term follow-up, as in previous reports [8]. Events occurring at any time point during follow-up require care.

Although there are various treatment methods for PAAs, our first-line surgical strategy was simple: open surgery with a medial approach and vein bypass grafting. However, the surgical management of the aneurysm sac varied among our patients (aneurysmectomy or ligation with bypass), and was determined by the location and size of the aneurysm (as well as the surgeon's judgment). Thus, we focused on the differences in the outcomes between these two management protocols. The rate of remnant sac enlargement after ligation with bypass was $32 \%$ - 35\% in previous reports [3] [13] [14]. However, there were only 3 cases (6.8\%) of sac enlargement in the present study. We assume that this difference in outcomes is related to differences in the ligation procedure. A bypass technique that involves only ligating the proximal and distal site of the PAA through two small incisions, as described by Edwards in 1969 [15], has been adopted through the years. In contrast, our modified "ligation" technique includes a partial resection and ligation of the branches, to the greatest extent possible. This may explain why we did not find any differences between the aneurysmectomy and ligation groups in terms of outcomes.

The comparison of ischemic symptoms between the aneurysmectomy and ligation groups did not reveal any specific findings or trends. None of the asymp- 
tomatic patients, or those with claudication, exhibited deteriorating symptoms, as we had expected. Notably, small diameter grafting was associated with a decrease in the ABI. Although the decrease was not critical in some patients, this finding demonstrates that a mismatch in the diameters may affect the ABI in some cases. Thus, it may be beneficial to select a prosthetic graft in cases in which a mismatch exists.

PAAs are known to be sometimes accompanied by concomitant aneurysms. Aneurysms in the abdominal aorta and contralateral popliteal artery are observed in $\sim 50 \%$ of PAA cases [8]. As the site of other aneurysms can vary from the subclavian artery to the lower limb artery, a routine imaging survey does not always cover the necessary range, resulting in a failure to note aneurysm lesions. An examination via palpation, which may now be regarded as obsolete, is necessary to complement imaging surveys.

In cases complicated by systemic diseases, the systemic disease may influence the surgical and follow-up strategies. We observed three cases of suspected Behçet's disease and one case of Klippel-Trenaunay syndrome in the present study. Approximately $2 \%$ of patients with Behçet's disease demonstrate vascular-related symptoms prior to diagnosis [16]. In the present study, none of the cases completely fulfilled the criteria for Behçet's disease; however, one patient with suspected Behçet's disease died during short-term follow-up and another required re-intervention twice, indicating a potential risk associated with this disease. Anastomotic complications are a notorious adverse event in Behçet's disease; long-term steroid or colchicine treatment should be prescribed [17]. Akagi et al. [18] reported a case of PAA in a patient with Klippel-Trenaunay syndrome. The authors suspected that the arterial malformation influenced arterial wall fragility; however, there was no supporting pathological evidence. Fortunately, we have not yet noted any such events in our case series. The outcomes of endovascular procedures, first developed in the 1990s, are reported to be equivalent to those of open surgery [19] [20] [21]. As open surgery is associated with a potential risk of stent fracture [22], it is not the first-line choice of treatment. In cases of Behçet's disease, which have an extremely high risk of anastomotic failure (particularly under inflammatory conditions), we assume that the endovascular procedure may be preferable to open surgery with anastomosis. Furthermore, clinicians should carefully observe juvenile patients with PAAs and those with systemic diseases, either diagnosed or suspected.

The present study has several limitations. The most critical limitation concerns the small sample size. A larger study sample would have allowed additional sub-analyses. However, the present study included patients from a single institution, operated on by a limited number of surgeons with similar skills, which may have reduced selection biases.

\section{Conclusion}

In the present study, we demonstrated the outcomes of open surgery for PAAs 
in a single Japanese institute. The patency rates were good, and our modified ligation with bypass procedure, which involves excising the sac as much as possible, exhibited less risk in terms of remnant sac enlargement compared to that in previous studies. Although there was no postoperative deterioration in the clinical symptoms of asymptomatic patients, graft mismatching resulted in a decrease in the ABI. In addition, Behçet's disease may be associated with a risk for postoperative adverse events.

\section{Disclosures}

All authors declare no conflicts of interest.

\section{References}

[1] Anton, G.E., Hertzer, N.R., Beven, E.G., O’Hara, P.J. and Krajewski, L.P. (1986) Surgical Management of Popliteal Aneurysms. Journal of Vascular Surgery, 3, 125-134. https://doi.org/10.1016/0741-5214(86)90075-3

[2] Whitehouse Jr., W.M., Wakefield, T.W., Graham, L.M., Kazmers, A., Zelenock, G.B., Cronenwett, J.L., et al. (1983) Limb-Threatening Potential of Arteriosclerotic Popliteal Artery Aneurysms. Surgery, 93, 694-699.

[3] Ebaugh, J.L., Morasch, M.D., Matsumura, J.S., Eskandari, M.K., Meadows, W.S. and Pearce, W.H. (2003) Fate of Excluded Popliteal Artery Aneurysms. Journal of Vas cular Surgery, 37, 954-959. https://doi.org/10.1067/mva.2003.258

[4] Ascher, E., Markevich, N., Schutzer, R.W., Kallakuri, S., Jacob, T. and Hingorani, A.P. (2003) Small Popliteal Artery Aneurysms: Are They Clinically Significant? Journal of Vascular Surgery, 37, 755-760. https://doi.org/10.1067/mva.2003.232

[5] Phair, A., Hajibandeh, S., Hajibandeh, S., Kelleher, D., Ibrahim, R. and Antoniou, G.A. (2016) Meta-Analysis of Posterior versus Medial Approach for Popliteal Artery Aneurysm Repair. Journal of Vascular Surgery, 64, 1141-1150. https://doi.org/10.1016/j.jvs.2016.05.064

[6] Zaraca, F., Ponzoni, A., Stingari, C., Ebner, J.A., Giovannetti, R. and Ebner, H. (2010) The Posterior Approach in the Treatment of Popliteal Artery Aneurysm: Feasibility and Analysis of Outcome. Annal of Vascular Surgery, 24, 863-870. https://doi.org/10.1016/j.avsg.2010.04.005

[7] Beseth, B.D. and Moor, W.S. (2006) The Posterior Approach for Repair of Popliteal Artery Aneurysms. Journal of Vascular Surgery, 43, 940-945. https://doi.org/10.1016/j.jvs.2005.12.062

[8] Huang, Y., Gloviczki, P., Noel, A.A., Sullivan, T.M., Kalra, M., Gullerud, R.E., et al. (2007) Early Complications and Long-Term Outcome after Open Surgical Treatment of Popliteal Artery Aneurysms: Is Exclusion with Saphenous Vein Bypass Still the Gold Standard? Journal of Vascular Surgery, 45, 706-715. https://doi.org/10.1016/j.jvs.2006.12.011

[9] Pulli, R., Dorigo, W., Troisi, N., Innocenti, A.A., Pratesi, G., Azas, L., et al. (2006) Surgical Management of Popliteal Artery Aneurysms: Which Factors Affect Outcomes? Journal of Vascular Surgery, 43, 481-487. https://doi.org/10.1016/j.jvs.2005.11.048

[10] Johnson 3rd, O.N., Slidell, M.B., Macsata, R.A., Faler, B.J., Amdur, R.L. and Sidawy, A.N. (2008) Outcomes of Surgical Management for Popliteal Artery Aneurysms: An Analysis of 583 Cases. Journal of Vascular Surgery, 48, 845-851. 
https://doi.org/10.1016/j.jvs.2008.05.063

[11] Mahmood, A., Salaman, R., Sintler, M., Smith, S.R., Simms, M.H. and Vohra, R.K. (2003) Surgery of Popliteal Artery Aneurysms: A 12-Year Experience. Journal of Vascular Surgery, 37, 586-593. https://doi.org/10.1067/mva.2003.141

[12] Davies, R.S., Wall, M., Rai, S., Simms, M.H., Vohra, R.K., Bradbury, A.W., et al. (2007) Long-Term Results of Surgical Repair of Popliteal Artery Aneurysm. European Journal of Vascular Endovascular Surgery, 34, 714-718. https://doi.org/10.1016/j.ejvs.2007.06.019

[13] Wakassa, T.B., Matsunaga, P., da Silva, E.S., Pinto, C.A., Kauffman, P., Aun, R., et al. (2006) Follow-up of the Aneurysmal Sac after Exclusion and Bypass of Popliteal Artery Aneurysms. Clinics, 61, 107-112. https://doi.org/10.1590/S1807-59322006000200004

[14] Ravn, H., Wanhainen, A. and Bjorch, M. (2007) Surgical Technique and Long-Term Results after Popliteal Artery Aneurysm Repair: Results from 717 Legs. Journal of Vascular Surgery, 46, 236-243. https://doi.org/10.1016/j.jvs.2007.04.018

[15] Edwards, W.S. (1969) Exclusion and Saphenous Vein Bypass of Popliteal Aneurysms. Surg Gynecol Obstet, 128, 829-830.

[16] Sarica-Kucukoglu, R., Akdag-Kose, A., Kayabali, M., Yazganoglu, K.D., Disci, R., Erzengin, D., et al. (2006) Vascular Involvement in Behçet's Disease: A Retrospective Analysis of 2319 Cases. International Journal of Dermatology, 45, 919-921. https://doi.org/10.1111/j.1365-4632.2006.02832.x

[17] Hosaka, A., Miyata, T., Hoshina, K., Okamoto, H., Shigematsu, K. and Oshima, A. (2014) Prognosis of Arterial Aneurysm after Surgery in Patients with Behçet's Disease. Int Angiol, 33, 419-425.

[18] Akagi, D., Ishii, S., Kitagawa, T., Nagawa, H. and Miyata, T. (2006) Popliteal Arterial Aneurysm Associated with Klippel-Trenaunay Syndrome: Case Report and Literature Review. Journal of Vascular Surgery, 43, 1287-1289. https://doi.org/10.1016/j.jvs.2006.02.022

[19] Leake, A.E., Avgerinos, E.D., Chaer, R.A., Singh, M.J., Makaroun, M.S. and Marone, L.K. (2016) Contemporary Outcomes of Open and Endovascular Popliteal Artery Aneurysm Repair. Journal of Vascular Surgery, 63, 70-76. https://doi.org/10.1016/j.jvs.2015.08.056

[20] Cervin, A., Tjarnstrom, J., Ravn, H., Acosta, S., Hultgren, R., Welander, M., et al. (2015) Treatment of Popliteal Aneurysm by Open and Endovascular Surgery: A Contemporary Study of 592 Procedures in Sweden. European Journal of Vascular Endovascular Surgery, 50, 342-350. https://doi.org/10.1016/j.ejvs.2015.03.026

[21] Golcherhr, B., Tielliu, I.F., Verhoeven, E.L., Mollenhoff, C., Antonello, M., Zeebregts, C.J., et al. (2016) Clinical Outcome of Isolated Popliteal Artery Aneurysms Treated with a Heparin-Bonded Stent Graft. European Journal of Vascular Endovascular Surgery, 52, 99-104. https://doi.org/10.1016/j.ejvs.2016.04.009

[22] Tielliu, I.F., Zeebregts, C.J., Vourliotakis, G., Bekkema, F., van den Dungen, J.J., Prins, T.R., et al. (2010) Stent Fractures in the Hemobahn/Viahahn Stent Graft after Endovascular Popliteal Aneurysm Repair. Journal of Vascular Surgery, 51, 1413-1418. https://doi.org/10.1016/j.jvs.2009.12.071 